TIFR/TH/97-02

hep-th/9702050

\title{
MICROSTATES OF NON-SUPERSYMMETRIC BLACK HOLES
}

\author{
Atish DabholkaR ${ }^{\dagger}$ \\ Tata Institute of Fundamental Research \\ Homi Bhabha Road, Mumbai, India 400005.
}

\begin{abstract}
A five-dimensional dyonic black hole in Type-I theory is considered that is extremal but non-supersymmetric. It is shown that the Bekenstein-Hawking entropy of this black hole counts precisely the microstates of a D-brane configuration with the same charges and mass, even though there is no apparent supersymmetric nonrenormalization theorem for the mass. A similar result is known for the entropy at the stretched horizon of electrically charged, extremal, but non-supersymmetric black holes in heterotic string theory. It is argued that classical nonrenormalization of the mass may partially explain this result.
\end{abstract}

February, 1997

$\dagger$ e-mail: atish@theory.tifr.res.in 


\section{Introduction}

The spectrum of supersymmetric states has played a crucial role in understanding strong coupling phenomena in string theory. A supersymmetric state (or a 'superstate' for short) is a state that preserves some of the supersymmetries, and belongs to a short representation of the supersymmetry algebra [1]. An important property of a superstate that follows from the supersymmetry algebra is that it is always extremal, i.e., its mass $M$ is always proportional to the absolute value of some charge $Z$. This also implies that a non-extremal state is always non-super, but the converse is not always true. In fact, we shall be interested in precisely such states, in Type-I and heterotic string theory, that are extremal but non-supert.

The significance of superstates to strong coupling physics stems from the fact that their exact spectrum can often be computed reliably in the semiclassical regime. One expects that, under suitable conditions, the number of states cannot jump discontinuously as we smoothly vary the coupling constant. In particular, a short multiplet at weak coupling is expected to remain short even at strong coupling. Moreover, because the extremality of a short multiplet is a consequence of the supersymmetry algebra, its mass is proportional to the charge even at strong coupling. With enough supersymmetries, the charge is sometimes not renormalized, which for the superstates implies that the mass is also not renormalized. This property of exact extremality is crucial for the stability of the superspectrum. Charge conservation together with energetic considerations are enough to ensure the stability of many superstates.

These special properties of the superspectrum have proved to be extremely useful recently, in particular, for obtaining a statistical interpretation in terms of the underlying microstates of the Bekenstein-Hawking entropy of certain supersymmetric black holes in string theory. In string theory, the spectrum of superstates is much richer than in field theory. For a given mass and charges, there is usually a large degeneracy of superstates which can be counted reliably at weak coupling. As one increases the strength of the

1 In recent literature a superstate is commonly called a BPS state. However, before the work of Witten and Olive [1], a BPS state meant a state that was only classically extremal. To distinguish between the two notions, we have used the terms 'super' and 'extremal' instead of 'BPS'. 
coupling, the superstate eventually undergoes a gravitational collapse, and forms a black hole. Because the spectrum of the superstates does not change as we vary the coupling, the degeneracy of the corresponding black-hole states at strong coupling must be the same as the degeneracy of superstates at weak coupling. This degeneracy has been shown to be in precise numerical agreement with the exponential of the Bekenstein-Hawking entropy in a large number of cases. In this paper, we shall be interested in a similar counting of states for a certain class of non-supersymmetric but extremal black holes that exist in toroidally compactified heterotic and Type-I string theory. In this case, supersymmetry alone does not protect the spectrum and there is no a priori reason to expect that the degeneracy at strong coupling should be the same as that at weak coupling. Surprisingly we still find this to be the case.

Our discussion of the non-supersymmetric states and the associated black holes will closely parallel that of the supersymmetric states. So, let us begin by recalling some facts about the superstates. One simple class of superstates are the perturbative, electric superstates in toroidally compactified heterotic string theory [2]. Consider heterotic string compactified on an $n$-dimensional torus to $D=10-n$ dimensions. There are 16 real supersymmetries corresponding to the 16 real components of a single Majorana-Weyl fermion in ten dimensions. All supersymmetries are carried by the right-movers. To discuss the spectrum it is convenient to use the Green-Schwarz formalism in the light-cone gauge. For a state of mass $M$ and charges $q_{L}$ and $q_{R}$ the Virasoro constraints are

$$
N_{L}=1+\alpha^{\prime}\left(\frac{1}{4} M^{2}-q_{L}^{2}\right), \quad N_{R}=\alpha^{\prime}\left(\frac{1}{4} M^{2}-q_{R}^{2}\right)
$$

where $N_{L}$, and $N_{R}$ are the number of transverse left-moving and right-moving oscillators respectively. A short representation corresponding to a superstate in spacetime that preserves 8 of the original 16 supersymmetries can be obtained only if all right-moving oscillators on the world-sheet are in the ground state, i.e. $N_{R}=0$. The mass-shell conditions (1.1) can then be written as:

$$
M^{2}=4 q_{R}^{2}, \quad N_{L}=1+\alpha^{\prime}\left(q_{R}^{2}-q_{L}^{2}\right)
$$

For large $N_{L}$, the degeneracy of these superstates $d\left(N_{L}\right)$ goes as $d\left(N_{L}\right) \sim e^{4 \pi \sqrt{N_{L}}}$. At large coupling, the superstate is described by a supersymmetric black hole. For these black 
holes the event horizon and the singularity coincide; so at first sight, the area $A$ of the event horizon and consequently the Bekenstein-Hawing entropy $S=A / 4$ appears to vanish. However, Sen [3] was able to show that, in four dimensions, if one computes the area at the stretched horizon, which is roughly string-length away from the event horizon, then the entropy is indeed proportional to the logarithm of the degeneracy of the perturbative superstates. Similar result holds for higher dimensions as well 《⿶.

Instead of considering the superstates that have $N_{R}=0$, let us now consider states that have $N_{L}=1$, but arbitrary $N_{R}$. The mass-shell condition now becomes

$$
M^{2}=4 q_{L}^{2}, \quad N_{R}=-\alpha^{\prime}\left(q_{R}^{2}-q_{L}^{2}\right)
$$

These states are still extremal at the classical level because the mass is proportional to $2\left|q_{L}\right|$, but they are no longer super because, for $N_{R} \neq 0$, they break all the right-moving supersymmetries, and belong to a long representation. At tree level, the perturbative states still have the Hagedorn degeneracy that goes as $d\left(N_{R}\right) \sim e^{\sqrt{N_{R}}}$. However, now supersymmetry no longer protects the mass formula, and in general the mass would be renormalized. Therefore, the degeneracy of states at strong coupling can be very different from this classical, perturbative formula. On the other hand, we can calculate the entropy at the stretched horizon for the corresponding extremal black holes as suggested in [5, 60. The calculation is identical to that of Sen for the supersymmetric black holes because it depends only on the low-energy bosonic fields which are insensitive to the orientation of the string apart from the labeling of the gauge fields. From [3], we see that in the normalizations of [3], the entropy is given by

$$
S_{B-H}=\frac{A}{4 G_{N}} \sim \frac{2 \pi C}{g} \sqrt{M^{2}-\frac{Q_{R}^{2}}{8 g^{2}}},
$$

where $m$ is the mass of the black hole in the Einstein metric, $Q_{L}$ is the charge, $g$ is the string coupling constant, and $C$ is a numerical constant. The statistical entropy calculated from the number of superstates is given by

$$
S_{\text {stat }}=\log d\left(N_{R}\right) \sim \sqrt{N_{R}} \sim \frac{1}{g} \sqrt{M^{2}-\frac{Q_{R}^{2}}{8 g^{2}}},
$$


after converting $M$ in (1.3) which is measured in the string metric and the charges $q_{L}$ to Sen's normalizations. Thus, the Bekenstein-Hawking entropy agrees, modulo a numerical coefficient, with the perturbative counting of states. Now, even though the bosonic fields are insensitive to the difference between left-moving and right-moving charges and oscillations, the fermionic fields and the supersymmetry transformations do notice this difference [7]. In particular, black hole solutions with $N_{R}=0$ preserve half the supersymmetries, whereas the black hole solutions with $N_{L}=1$ but $N_{R} \neq 0$ break all supersymmetries. We thus seem to obtain a surprising agreement between the Bekenstein-Hawking entropy and the perturbative counting of states for extremal but nonsupersymmetric black holes [5].6].

One of the disadvantages of purely electric extremal black holes is the need to consider the stretched horizon, which introduces the undetermined numerical coefficient $C$ in (1.5). In the supersymmetric case, this problem was overcome by considering dyonic black holes that have regular event horizon with nonzero area [8]. The Bekenstein-Hawking entropy is then simply given by a quarter of the horizon area which agrees precisely, without any undetermined numerical constant, with the microscopic counting of states. In this case, the microscopic superstates at weak coupling are not perturbative, but are given by a configuration of D-branes [9, 10]. The degeneracy of these states can be calculated at weak coupling using D-brane techniques.

Our objective will be to find non-supersymmetric but extremal black holes that also have a regular event horizon and nonzero area and compare it with the D-brane counting. Consider Type-I theory on $M^{5} \times S^{1} \times T^{4}$ where $M^{5}$ is the five-dimensional Minkowski spacetime with co-ordinates $\left(t, x_{1}, x_{2}, x_{3}, x_{4}\right), S^{1}$ is a circle of radius $R$ with co-ordinate $x_{5} \equiv x_{5}+2 \pi R$, and $T^{4}$ is a four-torus with co-ordinates $\left(x_{6}, x_{7}, x_{8}, x_{9}\right)$. Now, consider a configuration of $Q_{1}$ Dirichlet 1-branes wrapped around the circle, $Q_{5}$ Dirichlet 5-branes wrapped around the torus $S^{1} \times T^{4}$, and $n$ units of right-moving quantized momentum flowing along the circle. This particular configuration is motivated by the following reason. Consider the $S O(32)$ heterotic string compactified on a circle of radius $R$, and consider states that have winding number $m$ and momentum $n / R$ along the circle but no $S O(32)$ charges. For these states, we can take $q_{L}$ and $q_{R}$ appearing in (1.3) and (1.2) to be

$$
q_{L}=\left(\frac{n}{2 R}+\frac{m R}{2 \alpha^{\prime}}\right), \quad q_{R}=\left(\frac{n}{2 R}-\frac{m R}{2 \alpha^{\prime}}\right) .
$$


One can then consider either the superstates for which $N_{R}=0$ and $N_{L}=1-m n$, or the non-super but extremal states for which $N_{L}=1$ and $N_{R}=m n$. The important difference between the two cases is that because $N_{R}$ and $N_{L}$ are always non-negative, $m n$ is negative for the super-states but positive for the non-super-states. Thus, for a string that is wound with a positive orientation $(m>0)$, the superstates have left-moving momentum whereas the non-superstates have right-moving momentum. Under the duality between heterotic and Type-I theory [1], a fundamental, supersymmetric, winding string without oscillations is mapped onto the heterotic soliton string [12,13 which at weak coupling is described by the Dirichlet heterotic 1-brane [14]. An oscillating non-supersymmetric but extremal heterotic solitonic solution carrying right-moving momentum along the string with large and nonzero $N_{R}$ can be obtained as in [7, 15. Dimensional reduction of the oscillating solitonic string gives an electric, extremal black hole with vanishing horizon area. If we want a solution with nonzero area, we can add 5-branes. We are thus led to the configuration discussed above.

The dyonic state in Type-I theory couples only to the graviton $G_{M N}$ and the dilaton $\phi$ from the NS-NS sector, and the 2 -form potential $B_{M N}$ from the R-R sector. The low energy action for these fields is

$$
S=\frac{1}{16 \pi G_{10}} \int d^{10} x \sqrt{-G}\left(e^{-2 \phi}\left(R+4(\nabla \phi)^{2}\right)-\frac{1}{12} H^{2}\right) .
$$

The solution with three charges $Q_{1}, Q_{5}$, and $n$ is the same as the one that has been considered in Type-IIB theory before [16, 17, 18, 19, 20,21]. In the normalizations of [22] we have,

$$
\begin{gathered}
H=2 r_{5}^{2} \epsilon_{3}+2 r_{1}^{2} e^{-2 \phi} *_{6} \epsilon_{3}, \quad e^{-2 \phi}=\left(1+\frac{r_{5}^{2}}{r^{2}}\right)\left(1+\frac{r_{1}^{2}}{r^{2}}\right)^{-1} \\
d S^{2}=\left(1+\frac{r_{1}^{2}}{r^{2}}\right)^{-1 / 2}\left(1+\frac{r_{5}^{2}}{r^{2}}\right)^{-1 / 2}\left[-d t^{2}+d x_{5}^{2}+\frac{r_{n}^{2}}{r^{2}}\left(d t-d x_{5}\right)^{2}+\left(1+\frac{r_{1}^{2}}{r^{2}}\right) d x_{i} d x^{i}\right] \\
+\left(1+\frac{r_{1}^{2}}{r^{2}}\right)^{1 / 2}\left(1+\frac{r_{5}^{2}}{r^{2}}\right)^{1 / 2}\left[d r^{2}+r^{2} d \Omega_{3}^{2}\right]
\end{gathered}
$$

where $*_{6}$ is the Hodge dual in the six dimensions $x_{0}, . ., x_{5}, \epsilon_{3}$ is the volume element on the unit three-sphere, and $x^{i}, i=6, \ldots, 9$, are the co-ordinates of the torus with volume $(2 \pi)^{4} V$. 
This solution represents a black hole in the non-compact five dimensions. The parameters of the solution are related to the integral (positive) charges through the relations

$$
r_{1}^{2}=\frac{\lambda Q_{1} \alpha^{\prime}}{V}, \quad r_{5}^{2}=\lambda Q_{5} \alpha^{\prime}, \quad r_{0}^{2}=\frac{\lambda^{2} n \alpha^{\prime}}{R^{2} V}
$$

where $\lambda$ is the string coupling constant. If we take $r_{5}$ to be zero in this solution, then we simply obtain the heterotic solitonic string carrying momentum [7] but no oscillations. The supersymmetry of such a soliton has been discussed in detail in [7]. We only summarize the conclusions here. The term involving $\left(d t-d x_{5}\right)^{2}$ in the metric (1.9) corresponds to right-moving momentum along the soliton $(m n>0)$, and, as expected, the solution breaks all supersymmetries. If we replace this combination by $\left(d t+d x_{5}\right)^{2}$ instead, then the soliton carries left-moving momentum and preserves half the supersymmetries. Now, if we start with the nonsupersymmetric solution and add 5-branes, all supersymmetries still remain broken giving us the configuration we are interested in.

The Bekenstein-Hawking entropy and the energy of this black hole can be easily computed [20,21]:

$$
S_{B-H}=\frac{A_{10}}{4 G_{10}}=2 \pi \sqrt{Q_{1} Q_{5} n}, \quad E=\frac{R Q_{1}}{\lambda}+\frac{R V Q_{5}}{\lambda}+\frac{n}{R} .
$$

These expressions are the same for solutions carrying either right-moving or left-moving momentum. In particular, both non-supersymmetric and supersymmetric solutions are extremal.

We would now like to see if the counting of D-brane states at weak coupling can reproduce this entropy. In Type-I theory there are 32 9-branes in addition to the 1branes and the 5-branes. The open strings can end on any of these different type of branes. The $(1,1)$ strings with both ends on the 1-brane gives rise to the right-moving superstring, whereas the $(1,9)$, and $(9,1)$ strings give rise to left-moving current algebra of $\operatorname{Spin}(32) / \mathbb{Z}_{2}$ in the fermionic representation. These sectors are thus chiral. The sectors that are most relevant for the entropy counting are the $(1,5)$ and the $(5,1)$ strings. Recall that the Type-I theory is an orientifold of Type-IIB theory with orientifold projection $\frac{1+\Omega}{2}$ where $\Omega$ is the worldsheet parity. Under $\Omega$ the $(1,5)$ sector is identified with the $(5,1)$ sector which halves the number of states compared to Type-IIB theory. On the 
other hand, the 5-brane of Type-I with unit magnetic charge is really two 5-branes of Type-IIB put together [23,24], which doubles the number of states. So in the end we have effectively $4 Q_{1} Q_{5}$ superconformal free fields describing a superconformal sigma model on $\left(T^{4}\right)^{Q_{1} Q_{5}} / S\left(Q_{1} Q_{5}\right)$ exactly as in the Type-IIB case [8]. The counting of states is the same as that of right-moving oscillators at level $n$ in a conformal field theory with central charge $c=\frac{3}{2}\left(4 Q_{1} Q_{5}\right)$, which is given by $d(n)=e^{2 \pi \sqrt{c n / 6}}$. The statistical entropy is then

$$
S_{\text {stat }}=\log d(n)=2 \pi \sqrt{Q_{1} Q_{5} n},
$$

surprisingly, in precise agreement with (1.11).

The lack of supersymmetry of the black-hole solution when the momentum is rightmoving can be seen in the D-brane picture as well. The spacetime supersymmetries are only partially broken by a D-brane in the ground state and the unbroken supersymmetries give rise to the supersymmetries of the worldvolume theory [25]. The heterotic solitonic string is chiral and the unbroken spacetime supersymmetries give rise to the supersymmetry only in the right-moving sector. The ground state preserves these worldvolume supersymmetry but a state with nonzero right-moving energy breaks it completely. Consequently, for these states, the spacetime supersymmetry is also completely broken.

We should emphasize here that these black holes are not nearly supersymmetric but are far from being supersymmetric. There is no small parameter that measures the deviation from supersymmetry. The entropy of extremal and nonsupersymmetric black holes has been considered earlier in a different context [26] of rotating black holes in type-II theory in four and five dimensions, where a similar agreement was found with D-brane counting. In those examples it is the nonzero rotation that breaks supersymmetry completely even with extremality. Another example in four dimensions that has been considered before can be found in [27]. General black hole solutions in arbitrary dimensions that are supersymmetric but not extremal can be found in [28].

We do not fully understand how to explain this agreement between the counting of states at weak and strong coupling for these non-supersymmetric states. We present the following observations which may be relevant. The coupling constant of the theory and therefore the charges of these states are not renormalized by supersymmetry. It may be 
that the mass of these states is not renormalized for reasons other than supersymmetry. If this is true, then the states that are tagged by a particular mass and charges at weak coupling would continue to have the same mass and charges even at strong coupling, and then the counting can agree. It does appear that for the electric, perturbative states in heterotic string theory given by both (1.2) and (1.3), the mass is indeed not renormalized classically. Let us first see how one might define the notion of classical renormalization. There are three massless fields $G_{M N}, \phi$, and $B_{M N}$ that couple to the states. The classical renormalization can be defined as the sum of self-energies in these three fields. For the graviton there is no covariant stress-tensor but one can define the Landau-Lifshitz energymomentum pseudo-tensor $t_{L L}^{M N}$ [29]. The total conserved stress-tensor $\Theta^{M N}$ is then the sum of the stress-tensor for matter $T^{M N}$ and $t_{L L}^{M N}$. For a given metric that satisfies the Einstein equation, the total stress-tensor is

$$
\Theta^{P Q}=-\frac{1}{16 \pi G_{D}} \partial_{M} \partial_{N}\left[g\left(g^{P Q} g^{M N}-g^{P M} g^{Q N}\right)\right]
$$

where $g_{M N}$ is the Einstein metric with determinant $g$, and $G_{D}$ is Newton's constant in $D$ dimensions. We take spacetime to be a product of $(D-1)$ dimensional Minkowski spacetime and a circle of radius $R$. The radius can be quite small but still larger than the string scale. Let us now consider an oscillating heterotic string that wraps around this circle with right-moving oscillations. The solution for the massless fields describing such a state has been discussed in [7].15]. Let $(t, z)$ be the longitudinal coordinates and $y^{i}$, $i=1, \ldots,(D-2)$ the transverse coordinates. The dilaton $\phi$ satisfies the linear equation $\partial^{2}\left(e^{-2 \phi}\right)=-16 \pi \mu G_{D} \delta^{D-2}(y)$ where $\partial^{2}$ is the flat space Laplacian of the transverse co-ordinates, and $\mu$ is the bare string tension. The line-element in the Einstein metric is

$$
d s^{2}=e^{A}\left[-d t^{2}+d z^{2}+\vec{f} \cdot \vec{y}(d t-d z)^{2}\right]+e^{B}\left(d y^{i} d y^{i}\right)
$$

where $A=2(D-4) \phi /(D-2), B=-4 \phi /(D-2)$, and $\vec{f}$ is the profile of oscillation that depends only on $(t-z)$. For this time-dependent solution, all quantities like mass will, in general, be time-dependent. Here we consider the time-averaged mass. Substituting the metric in (1.13), and time-averaging over a period of oscillation, we obtain the total energy-density:

$$
\rho_{\text {total }}=-\frac{1}{16 \pi G_{D}} \partial^{2}\left(e^{-2 \phi}\right)=\mu \delta^{D-2}(y) .
$$


It is striking that the self-energy contributions due to various massless fields cancel precisely leaving only a delta-function source with the bare string tension $\mu$. The mass of these nonsupersymmetric but extremal electric states is therefore not renormalized classically if we consider only the massless fields. The massive fields can presumably be included in this analysis by adding the $\alpha^{\prime}$ corrections to the low-energy action for the massless fields.

Actually, for our purpose we do not really need a pointwise cancellation of self-energies as above. All that is needed is that the ADM mass be the same as the mass of a string source at the origin. For this purpose we need to match the solution onto string sources at the origin and then go to a coordinate system that is asymptotically flat. For the superstates (1.3) with left-moving oscillations, this is described in detail in $\S 2.6$ of [7], where it is shown that indeed there is no classical renormalization of the mass. Now, even for the non-super states, the classical calculation is the same. So we conclude that classically the mass of the extremal non-super states is also not renormalized. For the supersymmetric states, the classical nonrenormalization is a reflection of the nonrenormalization at the quantum level [2, 30]. It is very interesting to know, therefore, whether for the non-supersymmetric states also, the classical nonrenormalization of mass continues to hold at the quantum level [31]. It is tempting to speculate that there may be some hidden gauge invariance which can explain this nonrenormalization even without supersymmetry.

One final remark is that for a dyonic black hole with finite horizon area, or for the electric solutions carrying a pure momentum wave we do not know of an analogous result. Recall that a pure momentum wave without oscillations is obtained by replacing $\vec{f} \cdot \vec{y}$ in

(1.14) by $p / y^{D-4}$, but then the solution does not match onto a classical delta-function source [7]. In these cases, however, when there is an event-horizon, or when the solution does not match onto classical sources, it is not clear how to define the notion of bare mass or self-energy in a meaningful way to begin with.

\section{ACKNOWLEDGEMENTS}

I would like to thank Mike Duff, Ashoke Sen, and Lenny Susskind for useful discussions, Gary Horowitz, Jeff Harvey, and Juan Maldacena for comments on the draft, and the organizers of the 1996 Puri Workshop for inviting me to a very stimulating meeting where some of this work was done. 


\section{References}

[1] E. Witten and D. Olive, Phys. Lett. B78 (1978) 97.

[2] A. Dabholkar and J. A. Harvey, Phys. Rev. Lett. 63 (1989) 478.

[3] A. Sen, Mod. Phys. Lett. A10 (1995) 2081, hep-th/9504147.

[4] A. W. Peet, Nucl. Phys. B446 (1995) 211, hep-th/9504097.

[5] M. Duff, J. T. Liu, and J. Rahmfeld, hep-th/9612015.

[6] M. Duff and J. Rahmfeld, Nucl. Phys. B481 (1996) 332, hep-th/9605085.

[7] A. Dabholkar, J. P. Gauntlett, J. A. Harvey, and D. Waldram, Nucl. Phys. B474 (1996) 85, hep-th/9511053.

[8] A. Strominger and C. Vafa, Phys. Lett. B379 (1996) 99, hep-th/9601029.

[9] J. Polchinski, Phys. Rev. Lett. 75 (1995) 4724.

[10] J. Dai, R. Leigh, and J. Polchinski, Mod. Phys. Lett. A4 (1989) 2073;

P. Horava, Phys. Lett. B231 (1989) 251.

[11] E. Witten, Nucl. Phys. B443 (1995) 85, hep-th/9503124.

[12] A. Dabholkar, Phys. Lett. B357 (1995) 307, hep-th/9506160.

[13] C. M. Hull, Phys. Lett. B357 (1995) 345, hetp-th/9506194.

[14] J. Polchinski and E. Witten, Nucl. Phys. B460 (1996) 525, hep-th/9510169.

[15] C. G. Callan, J. M. Maldacena, and A. W. Peet, Nucl. Phys. B475 (1996) 631, hepth/9510134.

[16] A. Tseytlin, Mod. Phys. Lett. A11 (1996) 689, hep-th/9601177.

[17] M. Cvetic and A. Tseytlin, Phys. Rev. D53 (1996) 5619, hep-th/9512031.

[18] M. Cvetic and D. Youm, hep-th/9510098.

[19] F. Larsen and F. Wilczek, Phys. Lett. B375 (1996) 37, hep-th/9511064.

[20] G. Horowitz and A. Strominger, Phys. Rev. Lett. 77 (1996) 2368, hep-th/9602051.

[21] C. G. Callan and J. M. Maldacena, Nucl. Phys. B475 (1996) 645, hep-th/9602043.

[22] G. Horowitz, J. Maldaena, A. Strominger, Phys. Lett. B383 (1996) 151, hepth/9603109.

[23] E. Witten, Nucl. Phys. B460 (1996) 541, hep-th/9511030.

[24] E. Gimon and J. Polchinski, Phys. Rev. D54 (1996) 1667, hep-th/9601038.

[25] J. Huges and J. Polchinski, Nucl. Phys. B278 (1986) 147.

[26] G. Horowitz, D. Lowe, and J. Maldacena, Phys. Rev. Lett. 77 (1996) 430, hepth/9603195.

[27] D. M. Kaplan, D. A. Lowe, J. M. Maldacena, and A. Strominger, hep-th/9609204

[28] R. Khuri and T. Ortin, Phys. Lett. B373 (56) 1996, hep-th/9512178;

T. Ortin, hep-th/9612142.

[29] L. D. Landau and E. M. Lifshitz, The Classical Theory of Fields, 4th edition, Pergamon Press (1989), §96.

[30] A. Dabholkar, G. Gibbons, J. A. Harvey, and F. R. Ruiz-Ruiz, Nucl. Phys. B340 (1990) 33.

[31] A. Dabholkar, G. Mandal, and P. Ramadevi, work in progress 